\title{
Demonstrator influence on observer diet preference: Analyses of critical social interactions and olfactory signals
}

\author{
BENNETT G. GALEF, JR. and MONI STEIN \\ McMaster University, Hamilton, Ontario, Canada
}

\begin{abstract}
Previous studies have shown that interaction of an observer rat with a previously fed conspecific demonstrator enhances the observer's subsequent preference for the diet its demonstrator ate. The present series of experiments were undertaken to explore both the conditions sufficient to permit demonstrator influence on observer diet preference and the behavioral processes underlying such influence. We found (1) that an observer rat can be influenced in its subsequent diet selection by interaction for as little as $2 \mathrm{~min}$ with a demonstrator, (2) that during such brief interactions mouth-to-mouth contact between demonstrator and observer is necessary for demonstrator influence on observer diet preference, (3) that both cues emerging from the digestive tract of a rat fed by intragastric intubation and particles of food clinging to the fur of a demonstrator are sufficient to permit observers to identify their respective demonstrators' diets, (4) that exposure to a diet is effective in enhancing an observer's subsequent preference for that diet only if the diet is experienced in the presence of another rat, and (5) that diets experienced on the anterior of a live rat are more effective in altering observers' subsequent diet preferences than the same diets experienced either on the anterior of a dead rat or the posterior of a live one.
\end{abstract}

The results of a number of recent studies indicate that during social interaction olfactory cues pass from a recently fed rat (a demonstrator) to a naive conspecific (an observer), influencing that observer's subsequent diet selection. A naive rat that interacts with a demonstrator will, when given a choice of diets, exhibit a substantially enhanced preference for the diet its demonstrator ate (Galef \& Wigmore, 1983; Posadas-Andrews \& Roper, 1983; Strupp \& Levitsky, 1984).

Olfactory cues sufficient to produce demonstrator influence on observer diet preference are complex. Under at least some experimental conditions, simple exposure to a diet is not adequate to enhance preference for that diet, whereas exposure to the same diet in the presence of a rat will markedly enhance preference for it (Galef, Kennett, \& Stein, 1985; Strupp \& Levitsky, 1984; Experiment 4 , below). In such situations, it seems appropriate to think of the complex message passing from demonstrator to observer, resulting in alteration in observer diet preference, as consisting of two components: (1) a dietrelated component that permits an observer to identify its demonstrator's diet and (2) a rat-produced context that acts in concert with the diet-related component to enhance

This research was supported by Natural Sciences and Engineering Research Council of Canada Grant A0307 and a McMaster University Research Board Grant to B.G.G., Jr. We thank Sandra Vegeris for her outstanding assistance in the collection, analysis, and scoring of data and Harvey Weingarten and Mertice Clark for their thoughtful comments on earlier drafts. Moni Stein is now at the Medical School of the University of Toronto, Toronto, Ontario, Canada. Requests for reprints should be sent to Bennett G. Galef, Jr., Department of Psychology, McMaster University, Hamilton, Ontario, Canada L8S 4K1. an observer's subsequent preference for the diet of its demonstrator. Such segmentation of animal communications into a signal and context that jointly determine the response of a recipient to a communication has proven useful in previous analyses of communicative behaviors in animals (for a review, see Smith, 1977).

In the present series of experiments, we first explored the social interactions critical for demonstrator influence on subsequent observer diet preference (Experiments 1 and 2). We then examined the nature of the signals, both diet-related (Experiment 3) and contextual (Experiment 4), permitting demonstrators to influence the diet preferences of their respective observers.

\section{EXPERIMENT 1}

As a first step in investigating the social interactions permitting demonstrator influence on observer diet preference, we undertook a parametric study to determine the minimum duration of demonstrator-observer interaction sufficient to permit demonstrators to influence their respective observers' subsequent diet preferences. The briefer the period of social interaction we had to observe, the more carefully we could examine behavior during interaction to see if any particular behavioral acts were correlated with effective communication between demonstrator and observer.

\section{Method}

Subjects. The observers were 108 42-day-old Long-Evans rats obtained from Blue Spruce Farms (Altamont, NY). Prior to initiation of the experiment, all subjects were held in same-sex groups 


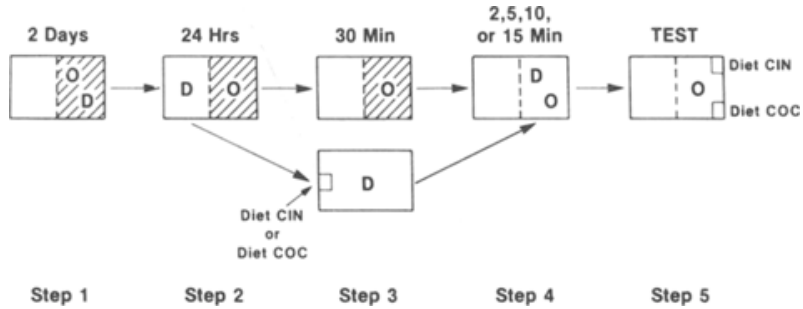

Figure 1. Schematic diagram of the procedure of Experiment 1. $\mathbf{O}=$ observer; $\mathbf{D}=$ demonstrator; hatching indicates that pellets of Purina Laboratory Rodent chow were present in the cage.

of 4 or 5 in $30 \times 30 \times 15 \mathrm{~cm}$ polypropylene cages and maintained on ad-lib Purina Laboratory Rodent chow and water.

One hundred and eight 90-110-day-old Long-Evans rats that had served as observers in previous experiments served as demonstrators in the present experiment. At the start of the experiment, each observer was paired randomly with a demonstrator of the same sex.

Apparatus. During the experiment, the subjects were housed and tested as demonstrator-observer pairs in $42.5 \times 24 \times 27.5 \mathrm{~cm}$ wiremesh hanging cages (Wahmann Co., Baltimore, MD). Each hanging cage was divided in two equal parts by a $1.25-\mathrm{cm}(1 / 2-$ in. $)$ screen partition $(24 \times 27.5 \mathrm{~cm})$ attached at the midpoints of each cage's 42.5-cm sides.

Procedure. Treatment of the subjects during the experiment was as follows (see Figure 1):

(1) Demonstrator and observer were maintained together with adlib access to Purina Laboratory Rodent Chow pellets for a 2-day period of familiarization with both apparatus and cagemate.

(2) Each demonstrator was moved to the side of the screen partition opposite from its observer and food-deprived for $24 \mathrm{~h}$ to ensure that the demonstrator ate when given the opportunity to do so.

(3) Chow was removed from each observer's side of the cage (in preparation for testing), and its demonstrator was moved to an enclosure in a separate room and allowed to feed for $30 \mathrm{~min}$ on either powdered Purina Laboratory Chow adulterated with $2 \%$ by weight Hershey's cocoa (cocoa-flavored diet) or powdered Purina Laboratory chow adulterated with $1 \%$ by weight McCormick's pure ground cinnamon (cinnamon-flavored diet).

(4) Each demonstrator was returned to its observer's cage, and demonstrator and observer were allowed to interact for $15,10,5$, or $2 \mathrm{~min}$.

(5) Each demonstrator was removed from the experiment, and the observer was offered, for $24 \mathrm{~h}$, two weighed food cups, one containing cinnamon-flavored diet and one containing cocoa-flavored diet.

\section{Results and Discussion}

The main results of Experiment 1 are presented in Figure 2, which shows the mean amount of cocoaflavored diet, as a percentage of total amount eaten, ingested by observers whose demonstrators ate either cinnamon- or cocoa-flavored diet during Step 3 of the experiment and interacted with their respective observers during Step 4 for $15,10,5$, or 2 min. As can be seen in Figure 2, those observers whose demonstrators ate cocoa-flavored diet ate a greater percentage of cocoaflavored diet than did those observers whose demonstrators ate cinnamon-flavored diet (Mann-Whitney U tests, see Figure 2 for $U$ and $p$ values).

Examination of Figure 2 also reveals a tendency for those observers that interacted for longer periods with their respective demonstrators to exhibit a greater tendency to ingest their respective demonstrators' diets. Statistical tests revealed that during testing (Step 5), ob-

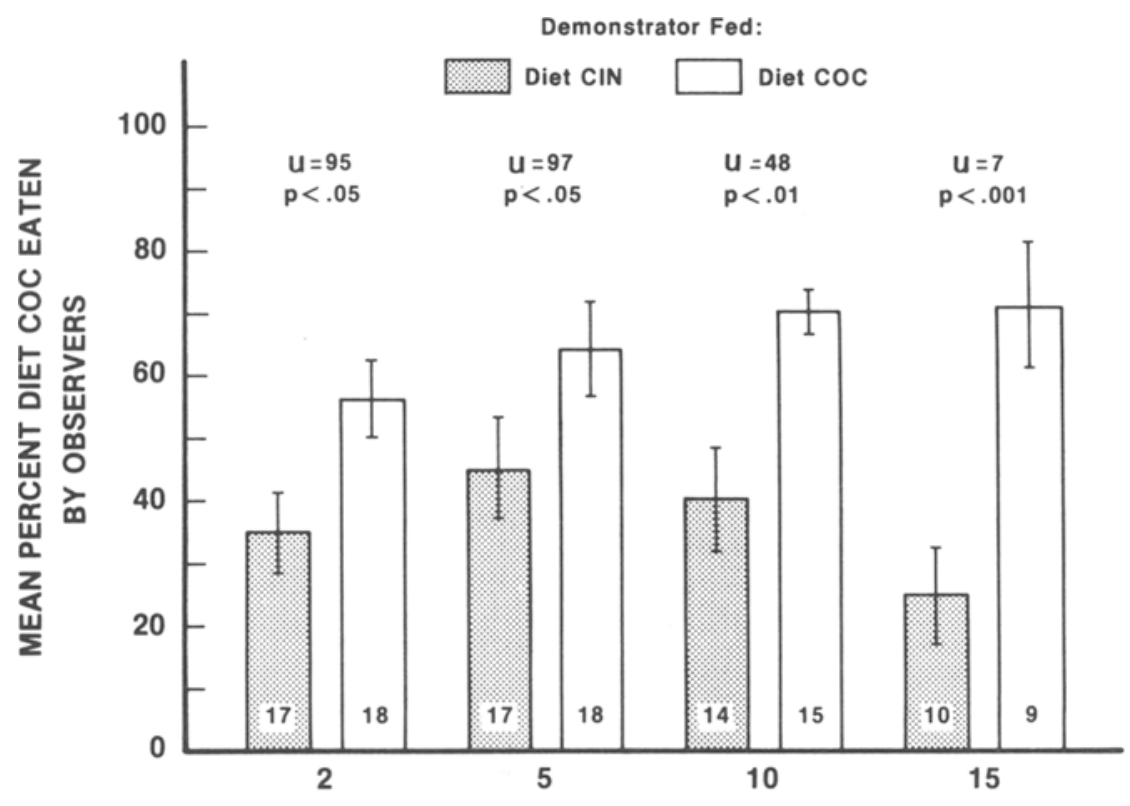

DURATION OF DEMONSTRATOR-OBSERVER INTERACTION (Min)

Figure 2. Mean amount of cocoa-flavored diet ingested, as a percentage of total amount eaten by observers whose demonstrators had eaten either cocoa- or cinnamon-flavored diet. Coc $=$ cocoa-flavored diet; $\mathrm{Cin}=$ cinnamon-flavored diet. The figure within each bar indicates the $n$ for that group. Flags indicate \pm 1 SE. 
servers that had interacted with their demonstrators (Step 4) for 15 min ingested a greater proportion of the diet that their respective demonstrators had eaten than did those observers that had interacted with their demonstrators for only 2 min [median test, $\chi^{2}(1)=3.98, p<.05$ ]. No other differences among groups in demonstrator influence on observer diet preference were significant.

Twenty-one of 35 observers that interacted with a demonstrator for 2 min exhibited a preference for their respective demonstrators' diets, and 17 of 19 observers that interacted with a demonstrator for $15 \mathrm{~min}$ did so $\left[\chi^{2}(1)=5.25, \mathrm{p}<.05\right]$. The finding that only $60 \%$ of observers exhibited a preference for their respective demonstrators' diets following a 2-min period of demonstrator-observer interaction suggests that, by watching demonstrators and observers during 2-min interactions, one might be able to identify behavioral events correlated with successful transmission of diet preference from demonstrator to observer.

\section{EXPERIMENT 2}

In the present experiment, we videotaped 2-min interactions between demonstrators and observers (Step 4 in Figure 1), then analyzed these interactions to see if we could identify behavioral events that differentiated observers that subsequently exhibited a preference for their respective demonstrators' diets from observers that failed to do so. To prevent our being misled either by observer bias or nonreplicable correlations, two independent groups of demonstrator-observer pairs were each scored blind by two independent observers.

\section{Method}

Subjects. Sixty-nine demonstrator-observer pairs served in two replications of the procedure described below-37 pairs in the first replicate, 32 pairs in the second. Demonstrator members of each pair were partially shaved so that they could be distinguished from observers on closed-circuit television during interaction.

Procedure. The procedure was the same as that described in the Method section of Experiment 1, except during Step 4 of the procedure (see Figure 1). In the present experiment, all demonstrators and observers were allowed to interact for $2 \mathrm{~min}$ in a $36 \times 24 \times$ $24 \mathrm{~cm}$ clear Plexiglas chamber with a $1.27-\mathrm{cm}(1 / 2 \mathrm{in}$.) screen floor. Behavior in the chamber was monitored by a black and white television camera mounted directly above the chamber and connected to a video tape recorder. A videotape of each 2-min interaction was independently reviewed by two scorers, both ignorant of the performance of observers during subsequent diet-preference testing (Step 5).

Each observer rat was scored in successive viewings of videotape as to: (1) whether or not it made mouth-to-mouth contact with its demonstrator, (2) the number of seconds it spent with its head in contact with its observer's head, and (3) the number of seconds it spent with its head in contact with some part of its demonstrator's body other than its head.

\section{Results}

In both Replicate 1 and Replicate 2, observers whose demonstrators ate cocoa-flavored diet ate a greater percentage of cocoa-flavored diet during testing (Step 5 of
Figure 1) than did observers whose demonstrators ate cinnamon-flavored diet (Replicate $1,62 \% \pm 10 \%$ vs. $38 \%$ $\pm 8 \%$; Replicate $2,59 \% \pm 7 \%$ vs. $38 \% \pm 6 \%$ ). MannWhitney U tests revealed these differences to be significant in Replicate $2(\mathrm{U}=77, \mathrm{p}<.05)$ and marginally so in Replicate $1(\mathrm{U}=111, \mathrm{p}=.06)$.

Review of the videotapes of the 2-min interactions of our 69 demonstrator-observer pairs revealed that interaction of observers with demonstrators did not occupy much of the time they were confined together in the apparatus (Step 4). Observers spent an average of $21.6 \mathrm{sec}$ with their heads in contact with or immediately adjacent to their respective demonstrators' heads or bodies (range $10-45.1 \mathrm{sec}$ ). The majority of this time was spent by the observers in investigating their demonstrators' bodies (mean $=16.1 \mathrm{sec}$, range $=5-43.1 \mathrm{sec}$ ); only brief periods were spent in investigating their heads and/or mouths (mean $=5.5 \mathrm{sec}$, range 2-13.1 sec). Observer rats appeared motivated to contact the ano-genital area of their respective demonstrators, actively pursuing and vigorously contacting the latter's nether regions. By contrast, contact with the demonstrators' heads and mouths seemed accidental, was brief in duration, lacked vigor, and was easily terminated by movement of the demonstrator.

Correlations between the amount of time individual observers spent in contact with the heads of their respective demonstrators during their interaction (Step 4) and the proportion of the demonstrators' diet eaten by those observers during testing (Step 5) were not significant (Replicate 1, Spearman's rho $=-.21$; Replicate 2, rho $=.04$ ). Similarly, correlations between the time spent by observers contacting the posterior portions of their respective demonstrators and their tendency to eat the same diet as those demonstrators were not significant (Replicate 1, rho $=-.11$; Replicate 2, rho $=-.16$ ) .

As can be seen in Figure 3, observers' contacts with the mouths of demonstrators proved to be a predictor of their subsequent preference for their respective demonstrators' diets. In both Replicate 1 and Replicate 2, those observers that were scored as having contacted the mouths of their demonstrators showed a significant tendency to eat the same diet as their respective demonstrators; those observers scored as not contacting the mouths of their demonstrators failed to exhibit a preference for their respective demonstrators' diets.

Interscorer reliabilities were: for mouth-to-mouth contact, .86; for length of observers' time in contact with the bodies of demonstrators, .85; and for length of observers' time in contact with the heads of demonstrators, .91. All analyses reported above were based on scores assigned by a single scorer, S.V.

\section{Discussion}

Posadas-Andrews and Roper (1983, p. 267), in an experiment similar to the present one, observed that "when a [fed] rat was returned to the home cage ... it was vigorously investigated by other animals, especially in its 


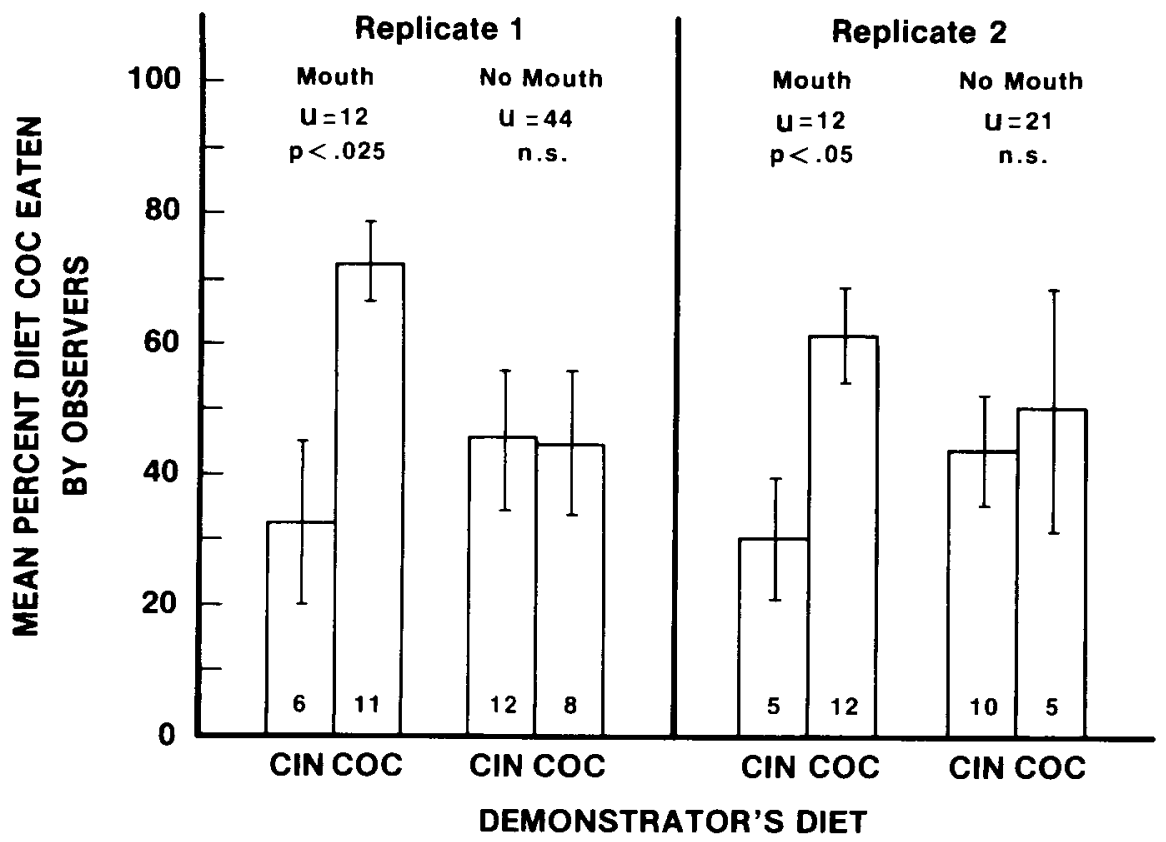

Figure 3. Mean amount of cocoa-flavored diet ingested, as a percentage of total amount eaten, by observers whose demonstrators had eaten either cocoa-flavored or cinnamon-flavored diet. The figure presents separately data from observers in two replicates that either made or failed to make mouth-to-mouth contact with their respective demonstrators. Coc $=$ cocoa-flavored diet; Cin = cinnamon-flavored diet; bars $= \pm 1 \mathrm{SE}$. Numbers in histograms $=$ n/group.

facial region. Quite often the attentions of the other rats were so persistent that [the returned rat] was forced to lie on its back while the others sniffed, pawed and groomed it. Given that the test food [in Posadas-Andrews and Roper's experiments] was of a paste-like consistency, it seemed likely that particles of food were adhering to the leader's [demonstrator's] face and paws, and that these provided information about the nature of the test diet."

None of the 69 observers studied in the present experiment exhibited vigorous or persistent exploration of the facial region of its demonstrator. Possibly this difference in behavior during the interactions of demonstrators and observers in the Posadas-Andrews and Roper study and our own resulted from Posadas-Andrews and Roper's use both of observers that had been food-deprived for $7 \mathrm{~h}$ prior to their interactions with demonstrators and of a sticky food that was likely to cling to the fur of demonstrators. (Our observers were satiated at the time of interaction and our demonstrators ate a dry, powdered food.) In any case, our finding of a correlation between an observer's preference for the demonstrator's diet and mouth-to-mouth contact between the observer and demonstrator is consistent with Posadas-Andrews and Roper's suggestion that particles of food adhering to the faces of demonstrators might provide information about the nature of the diet a demonstrator had previously eaten.

It is, of course, also possible, as Posadas-Andrews and Roper (1983) suggested, that observers are informed as to the diets their respective demonstrators had eaten while at a distance from the demonstrators, and that mouth-tomouth observer-demonstrator contact provides the contextual cues which cause observers to subsequently prefer their respective demonstrators' diets. There is some reason to credit this second interpretation. As Figure 3 reveals, Replicate 2 observers were more likely to make mouthto-mouth contact with demonstrators that had eaten the cocoa-flavored diet than with demonstrators that had eaten the cinnamon-flavored diet. Twelve of 17 observers that interacted with demonstrators fed the cocoa-flavored diet made mouth-to-mouth contact with their demonstrators, whereas only 5 of 15 observers that interacted with demonstrators fed the cinnamon-flavored diet did so $\left[\chi^{2}(1)\right.$ $=4.5, \mathrm{p}<.05]$. The results of Replicate 1 were in the same direction, but were not significant $\left[\chi^{2}(1)=2.35\right.$, $.1<\mathrm{p}<.2$ ]. If, in fact, observers are more likely to make mouth-to-mouth contact with demonstrators fed the cocoa-flavored diet, then they must be able to distinguish, from a distance, demonstrators fed the cocoa-flavored diet from those fed the cinnamon-flavored diet.

We have previously shown that during a 15 -min period of interaction, demonstrators can influence observers' subsequent diet selections even if the demonstrators and observers are denied the opportunity for physical contact (see also Posadas-Andrews \& Roper, 1983). Demonstrators, anesthetized and layed out for 15 min with their noses $2.5 \mathrm{~cm}$ from a screen that restrained observers, can still influence their respective observers' subsequent diet preferences (Galef \& Wigmore, 1983, Experiment 4). 
The results of the present experiment suggest that during a 2-min interaction, mouth-to-mouth contact may be necessary for a demonstrator to influence a subsequent observer diet preference. Taken together, the present findings and those reported in Galef and Wigmore (1983) lead us to suggest that there may be two types of interaction, each sufficient to allow demonstrators to influence observers' subsequent diet preferences. Mouth-to-mouth contact may provide the opportunity for rapid communication of information from demonstrator to observer. Interaction at a distance may require longer periods for diet identification or for contextual information to pass from demonstrator to observer.

\section{EXPERIMENT 3}

As mentioned in the introduction to the present paper, the results of our previous studies suggest that two types of signal are necessary to permit demonstrator influence on observer diet preference: first, an olfactory signal adequate to identify the demonstrator's diet (Galef \& Wigmore, 1983), and, second, a cue produced by a demonstrator providing the sensory context within which the diet-identifying signal will affect the observer's subsequent behavior (Galef, Kennett, \& Stein, 1985; Strupp \& Levitsky, 1984; see also Experiment 4, below). In the present experiment, we continue our examination of the first of these signals, that identifying the demonstrator's diet. In Experiment 4, we explore the nature of the contextual cues, emitted by demonstrator rats, that combine with diet-related cues to effect a subsequent observer diet preference.

In a recent study (Galef et al., 1985, Experiment 4), we found that following interaction with an anesthetized demonstrator, observers exhibited a preference for the powdered diet that had been applied to the face of that demonstrator. Similarly, Posadas-Andrews and Roper (1983) have interpreted their data as indicating that a diet applied to the fur of a demonstrator is sufficient to affect an observer's subsequent diet selection. Such findings indicate that ingestion of a diet by a demonstrator is not a necessary condition for demonstrator influence on observer diet preference. In the present experiment, we sought to determine whether food residing in a demonstrator's digestive tract could also produce a signal that would allow the observer to identify its demonstrator's diet.

\section{Method}

Subjects. Forty 42-day-old Long-Evans rats obtained from Blue Spruce Farms served as observers, and an additional 40 90-dayold rats from the same source served as demonstrators.

Apparatus. During the period of interaction between demonstrator and observer (Step 4 of Figure 1), the observer and demonstrator were placed in an apparatus (illustrated in Figure 4) constructed from a 2.45-liter (15.2-cm-high, 19.0-cm-top-diam, 14.0-cmbottom-diam) cardboard bucket (Lily-Tulip Inc., Toledo, $\mathrm{OH}$ ) of the type commonly used by fast-food franchises.

A circular opening ( $5-\mathrm{cm}$ diameter) was cut in the bucket wall $12 \mathrm{~cm}$ above its floor. Through this hole was inserted, for half its length, a 16-cm-long, $5-\mathrm{cm}$-diam tube constructed of .63-cm $(1 / 2-$ in.) screen. The end of this tube inside the bucket was closed with a disk of screen; the end outside the bucket was left open. A tightfitting cardboard lid served to prevent observers from leaving the bucket.

Procedure. Treatment of the subjects during the experiment was similar to that of Experiment 1 (see Figure 1 and Method of Experiment 1) with the following exceptions:

Step 2: The demonstrators were deprived of both food and water for 1 day.

Step 3: Twenty of the demonstrators were allowed access for $30 \mathrm{~min}$ to one of two solutions, either coffee-flavored water $(2.1 \%$ wt/vol Sanka Decaffeinated Instant Coffee in water) or vinegarflavored water $(3.2 \%$ vol/vol Allen's Pure Apple Cider Vinegar in water). Immediately following the end of the 30-min drinking period, each demonstrator was anesthetized (intraperitoneal injection of $50 \mathrm{mg} / \mathrm{kg}$ of sodium pentobarbital) and placed in the screen cylinder of the apparatus illustrated in Figure 4.

The remaining 20 demonstrators were anesthetized (intraperitoneal injection of $50 \mathrm{mg} / \mathrm{kg}$ sodium pentobarbital) and then stomach loaded, using a size 8 feeding tube (Bard Canada Inc., Mississauga, Ont.), with $1.5 \mathrm{cc}$ of either the vinegar- or coffee-flavored solution, to which a small amount of green food coloring had been added to facilitate detection of any regurgitated fluids. After stomach loading, these demonstrators were also placed in the screen cylinder of the apparatus illustrated in Figure 4.

Step 4: Each observer was placed in a bucket and allowed to interact with an anesthetized demonstrator for $30 \mathrm{~min}$.

Step 5: The demonstrators were removed from the experiment, and each observer was offered the choice between coffee- and vinegar-flavored solutions for $24 \mathrm{~h}$.

\section{Results}

The main results of Experiment 3 are presented in Figure 5, which shows the mean amount of coffeeflavored solution, as a percentage of total amount ingested, imbibed by observers whose demonstrators had drunk or been stomach-loaded with either vinegar- or coffeeflavored solution. As can be seen in the figure, observers whose demonstrators had been stomach-loaded with coffee-flavored solution drank a greater percentage of coffee-flavored solution than did the observers whose demonstrators had been stomach-loaded with vinegarflavored solution (Mann-Whitney $U=13, p<.01$ ). Similarly, the observers whose demonstrators drank coffee-flavored solution exhibited an enhanced preference for that solution relative to those observers whose demon-

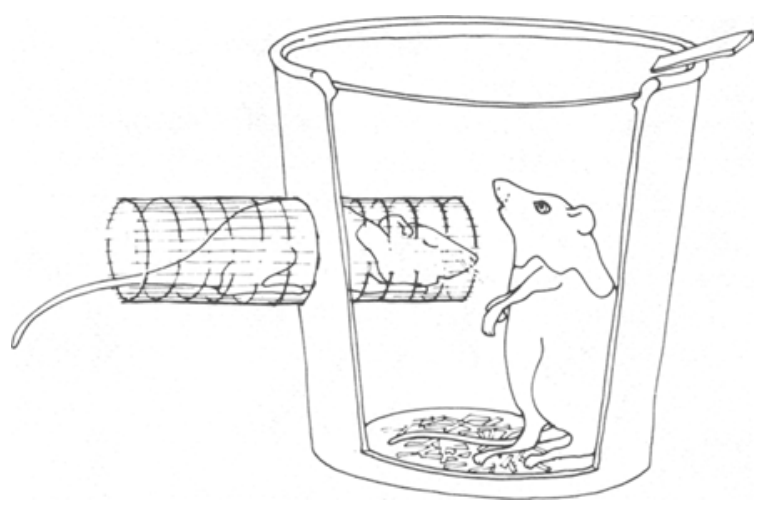

Figure 4. Apparatus used in Experiments 3 and 4. 


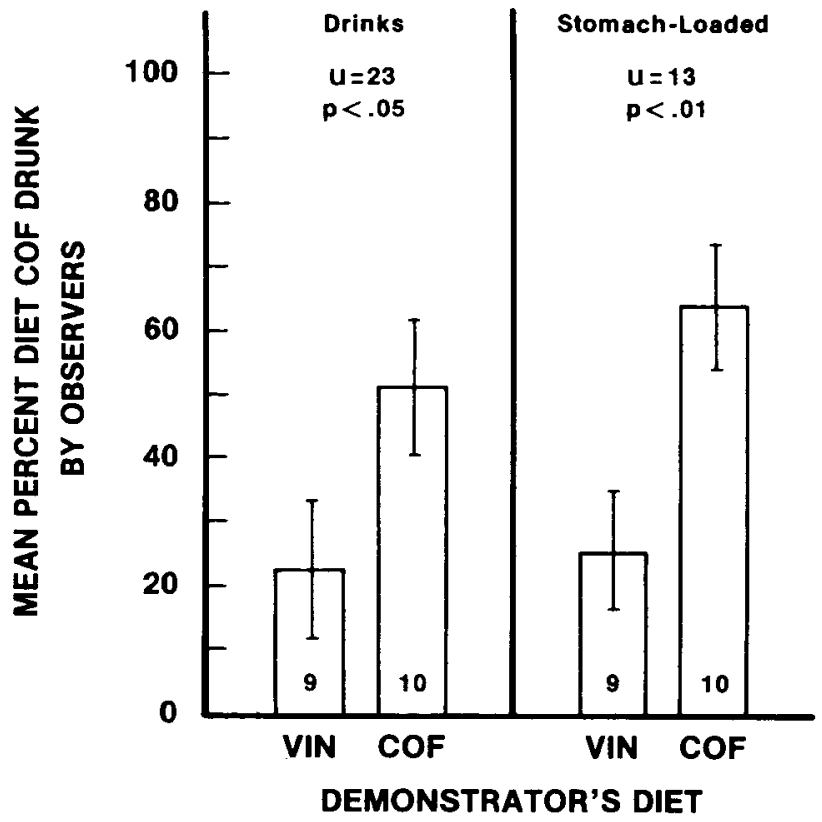

Figure 5. Mean amount of coffee-flavored solution, as a percentage of total amount drunk, ingested by observers whose demonstrators had drunk or been stomach loaded with vinegar- or coffeeflavored solutions. Vin = vinegar-flavored solution; Cof $=$ coffeeflavored solution; bars $= \pm 1$ SE.

strators drank vinegar-flavored solution (Mann-Whitney $\mathrm{U}=23, \mathrm{p}<.05$ ). The magnitude of observer preference for their respective demonstrators' solutions did not differ between observers whose demonstrators had drunk the solutions and those whose demonstrators had been tube-fed (Mann-Whitney $\mathrm{U}=148, \mathrm{p}=$ n.s.).

\section{Discussion}

The finding that demonstrator rats that receive a relatively small stomach load of flavored water increase their observers' preference for liquids of that flavor indicates that demonstrator-diet identification by observers does not depend on observer exposure to samples of diet exposed on the surface of the demonstrator. Taken together with the finding that samples of diet clinging to the face of an anesthetized demonstrator are adequate to permit observer identification of their respective demonstrators' diets (Galef et al., 1985; Posadas-Andrews \& Roper, 1983), the results of the present study suggest that either of two types of diet-identifying signals is sufficient to permit observer identification of demonstrators' diets. Both diet samples exposed on the surface of a demonstrator and dietidentifying cues emerging from the digestive tract of a demonstrator are sufficient to provide observers with information that permits observer identification of demonstrator's diet.

\section{EXPERIMENT 4}

In the present experiment, demonstrators were exposed to the same diet-identifying cues in a variety of contexts to permit identification of critical contextual features that would permit the demonstrator to influence the observer's subsequent diet preference. The studies described below were conducted as nine separate experiments over a 10month period. In the interests of brevity, all are described as nine studies in a single experiment. Each of the nine studies contained one group of observers that interacted with "powdered-face" demonstrators (our control condition, described below) and one or more groups of observers that interacted with demonstrators treated in some other way (see Procedure below).

\section{Method}

Subjects. Three hundred and fifty-two 42-day-old Long-Evans rats received from Blue Spruce Farms served as observers. An additional 271 90-104-day-old rats, observers in previous experiments, served as demonstrators.

Procedure. The procedure of the present experiments was similar to that of Experiment 3, except in the treatment of demonstrators during Steps 3 and 4 (see Figure 1 and Method of Experiment 3) and the diets offered observers during diet-preference testing (Step 5 of Figure 1). In the present experiment, during testing, all observers were offered a choice between cinnamon- and cocoa-flavored diets, the composition of which is described in the Method section of Experiment 1. Furthermore, all demonstrators in the present experiment were exposed to either cinnamon- or cocoa-flavored diets during Step 3. The various experiments differed in the way in which the diets were applied to demonstrators (Step 3 of Figure 1) and the way in which demonstrators were presented to observers during demonstrator-observer interaction in the apparatus illustrated in Figure 4. The treatment of various groups of demonstrators during Steps 3 and 4 of Figure 1 is described below.

Powdered-face demonstrators ( 9 replicates, 152 demonstrators, 152 observers). Powdered-face demonstrators were anesthetized (intraperitoneal injection $50 \mathrm{mg} / \mathrm{kg}$ sodium-pentobarbital) and their faces rolled in either cinnamon- or cocoa-flavored diet. Each demonstrator was then placed in the cylinder of the apparatus illustrated in Figure 4 with its head inside the bucket, as illustrated in the figure.

Powdered-rear demonstrators ( 3 replicates, 62 demonstrators, 62 observers). Powdered-rear demonstrators were treated exactly like powdered-face demonstrators except that: (1) following anesthetization, the hindquarters of each demonstrator were rolled in either cinnamon- or cocoa-flavored diet and (2) each powderedrear demonstrator was placed in the cylinder of the apparatus illustrated in Figure 4 with its rear inside the bucket and its head outside the bucket.

Dead powdered-face demonstrators ( 3 replicates, 57 demonstrators, 57 observers). Dead powdered-face demonstrators were treated like powdered-face demonstrators except that they were given a lethal overdose of sodium pentobarbital $(150 \mathrm{mg} / \mathrm{kg})$ rather than an anesthetic dose.

Surrogate demonstrators (4 replicates, 0 demonstrators, 81 observers). Observers in this group interacted during Step 4 with a rat-size surrogate demonstrator. This surrogate was constructed of cotton batting stuffed into a length of seamless tubular gauze (Size 12 Tubegauz, Scholl Canada Inc., Toronto, Ont.) that had been stapled closed at one end. The closed end of the surrogate was rolled in either cinnamon- or cocoa-flavored diet, and the surrogate was introduced into the screen cylinder of the apparatus illustrated in Figure 4 with the closed end inside the bucket.

Data presentation. In order to present the very large amount of data collected in a single figure, we devised a summary descriptive statistic for each of the nine studies conducted. We divided the mean percentage cocoa-flavored diet eaten by observers whose demonstrators (or surrogates) were powdered with cocoa-flavored diet by the mean percentage cocoa-flavored diet eaten by observers whose demonstrators (or surrogates) were powdered with cinnamon- 
flavored diet, to produce a cocoa-demonstrator/cinnamondemonstrator ratio. In those cases in which the cocoademonstrator/cinnamon-demonstrator ratio $>1$, the observers tended to eat the same diet as their respective demonstrators; in those cases in which the cocoa-demonstrator/cinnamon demonstrator ratio $\leq 1$, the observers did not tend to eat the same diet as their respective demonstrators. To determine the statistical significance within studies of differences in the percentage of cocoa-flavored diet eaten during testing (Step 5 of Figure 1) by observers whose demonstrators had been powdered with either cocoa- or cinnamonflavored diet, we applied the Mann-Whitney $U$ test to the percentage cocoa-flavored diet eaten by observers in the two groups.

\section{Results}

The main results of Experiment 4 are presented in Figure 6, which shows the cinnamon-demonstrator/cocoademonstrator ratios for each of the nine studies conducted. As examination of the figure reveals, observers interacting with powdered-face demonstrators consistently exhibited a statistically significant tendency to choose their respective demonstrators' diets for ingestion; in nine of nine replicates, the observers whose demonstrators had been powdered with cocoa-flavored diet ate a significantly greater proportion of that diet than did observers whose demonstrators had been powdered with cinnamon-flavored diet (Mann-Whitney $U$ tests, see Figure 5 for $p$ values).

Observers interacting with a diet-powdered surrogate failed to exhibit a tendency to match their diet selections to the diet placed on their respective surrogates; in three of four replicates, the observers exhibited cocoademonstrator/cinnamon-demonstrator ratios of less than 1 (indicating a tendency for observers to avoid eating the diet with which their respective surrogates had been powdered), whereas subjects in the fourth replicate exhibited a nonsignificant tendency to choose for ingestion the diets placed on their respective demonstrators. A comparison of the percentage of demonstrators' diets eaten by the 81 observers that had interacted with surrogates and the percentage of demonstrators' diets eaten by the 77 concurrently run observers that had interacted with powderedface demonstrators revealed that observers of powderedface demonstrators ingested significantly more of their respective demonstrators' diets than did observers of surrogates $[t(156)=3.91, p<.001]$. These data corroborate our previous findings that: (1) diets presented on a rat demonstrator are more effective in altering observer diet preference than are diets presented on a surrogate, and (2) simple exposure to a diet is not sufficient to enhance observers' preference for it (Galef et al., 1985; see also Strupp \& Levitsky, 1984).

A comparison of the percentage of demonstrators' diets eaten by the 57 observers that had interacted with deadpowdered-face demonstrators and the percentage of demonstrators' diets eaten by the 52 concurrently run observers that had interacted with powdered-face demonstrators revealed that the latter observers ingested significantly more of their respective demonstrators' diets than did the former $[t(107)=1.73, p<.05]$. Similarly, the 62 observers that had interacted with powdered-rear demonstrators ingested significantly less of their respective demonstrators' diets than the 46 concurrently run observers that had interacted with powdered-face demonstrators $[\mathrm{t}(106)=2.02, \mathrm{p}<.05]$.

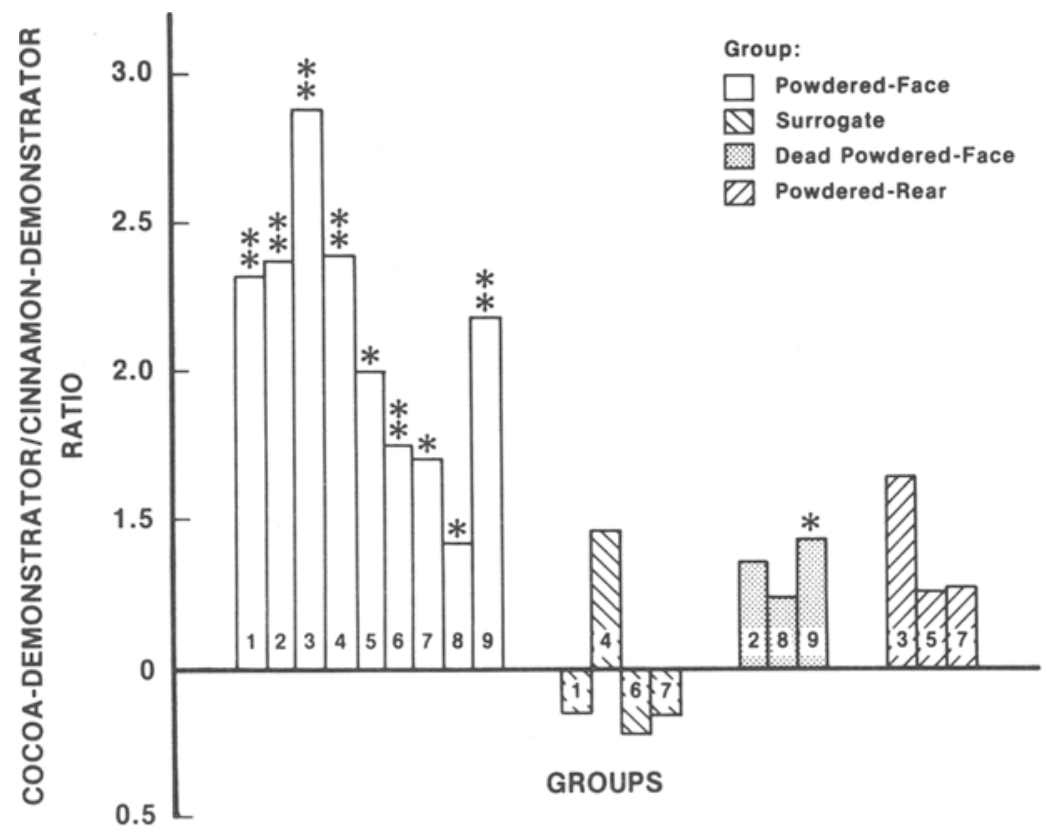

Figure 6. Cocoa-demonstrator/cinnamon-demonstrator ratios of 19 experimental groups. (See method of Experiment 4 for explanation of ratio and groups.) Subjects were assigned randomly across groups with the same number. ${ }^{*} p<.05,{ }^{* *} p<.01$, by Mann-Whitney U test. 
A comparison of diet selection by observers that had interacted with surrogates $(n=81)$ with that of observers that had interacted either with powdered-rear and deadpowdered-face demonstrators $(n=129)$ revealed that observers that had interacted with dead-powdered-face or powdered-rear demonstrators ingested a greater percentage of their respective demonstrators' diets than did the observers that had interacted with surrogates $[t(198)=$ $2.63, \mathrm{p}<.01]$. These analyses indicate that although both the anterior of dead demonstrator rats and the posterior of live demonstrator rats provide contextual cues that render a diet attractive to observers, these contextual cues are not as effective as those emerging from the anterior of a live demonstrator.

\section{GENERAL DISCUSSION}

The picture that emerges from the results of the present experiments, considered together with the results of previous work on inter-rat communication of information concerning distant diets, is a fairly complex one. First, the effects of demonstrators on the subsequent food preferences of observers can depend on more than simple exposure of observers to diet-identifying cues emitted by demonstrators (Galef et al., 1985; Experiment 4, above). Rat surrogates powdered with a diet failed to affect their observers' subsequent diet preferences, whereas anesthetized rats powdered with a diet had marked effects on observers' subsequent diet preferences. Similarly, Strupp and Levitsky (1984, Experiment 2) have found that the presence of a conspecific is necessary if exposure of a rat to diet-related cues is to result in an enhanced preference for that diet. On the other hand, Posadas-Andrews and Roper (1983), using procedures similar to those described in the present paper, have reported data they interpret as showing that simple exposure of naive rats to the odor of a diet is in itself sufficient to enhance a preference for that diet. Unfortunately, the data analyses Posadas-Andrews and Roper published are not adequate to support that contention, so one does not know whether a comparison of the details of our procedures and those of Strupp and Levitsky (1984) with those of PosadasAndrews and Roper might provide some insight into the conditions under which simple exposure to diet-related cues does or does not result in an enhanced preference for a diet. Review of the literature suggests both that (1) exposure to a particularly salient odor (such as garlic) enhances a subsequent preference for diets adulterated with the salient odor (Bronstein \& Crockett, 1976), and (2) amount of intake of a single diet is more effective than a choice procedure in revealing effects of simple exposure on subsequent diet preference (Domjan, 1976, 1977).

Unfortunately, the range of circumstances under which simple exposure to a diet enhances preference for that diet is poorly defined. Hence, the role of simple exposure to diet-related cues in demonstrator influence on observer diet preference must be determined in each individual case. The results of the present Experiment 4, of Galef et al. (1985), and of Strupp and Levitsky (1984) all strongly suggest that in circumstances in which simple exposure to a diet is not sufficient to enhance a preference for that diet, exposure of a naive rat to the same diet within the context provided by the presence of a conspecific will result in an enhanced preference for that diet.

Second, the diet-related cues emitted by demonstrators can emerge from either of two sources: particles of food clinging to the exterior of a demonstrator (as suggested by Posadas-Andrews \& Roper, 1983) or samples of food residing in the digestive tract of demonstrators (Experiments 3 and 4, above). Third, although the contextual cues resulting in enhancement of observer preference for demonstrators' diets seem to have a number of sources, the most potent of these cues seem to emerge from the anterior of live rats (Experiment 4). Last, during 2-min periods of interaction between demonstrator and observer, mouth-to-mouth contact appears critical for successful demonstrator influence on subsequent observer diet preference. There is some reason to believe both that it is the contextual cues that are transmitted during mouthto-mouth contact and that, during longer periods of demonstrator-observer interaction, mouth-to-mouth contact may not be necessary for demonstrator influence on subsequent observer diet preference (Galef \& Wigmore, 1983; Experiments 2 and 4 above).

\section{REFERENCES}

Bronstein, P. M., \& Crockett, D. P. (1976). Exposure to the odor of food determines the eating preference of rat pups. Behavioral $B i$ ology, 18, 387-392.

Domjan, M. (1976). Determinants of the enhancement of flavored water intake by prior exposure. Journal of Experimental Psychology: Animal Behavior Processes, 2, 17-27.

DomJAN, M. (1977). Attenuation and enhancement of neophobia for edible substances. In L. M. Barker, M. R. Best, \& M. Domjan (Eds.), Learning mechanisms in food selection. Waco, TX: Baylor University Press.

Galef, B. G., JR., Kennett, D. J., \& Stein, M. (1985). Demonstrator influence on observer diet preference: Effects of simple exposure and the presence of a demonstrator. Animal Learning \& Behavior, 13, 25-30.

GaleF, B. G., JR., \& Wigmore, S. W. (1983). Transfer of information concerning distant foods: A laboratory investigation of the "information centre' hypothesis. Animal Behaviour, 31, 748-758.

Posadas-Andrews, A., \& Roper, T. J. (1983). Social transmission of food-preferences in adult rats. Animal Behaviour, 31, 265-271.

SмIтн, W. J. (1977). The behavior of communicating. Cambridge, MA: Harvard University Press.

StrupP, B. J., \& Levitsky, D. A. (1984). Social transmission of food preference in adult hooded rats (Rattus norvegicus). Journal of Comparative Psychology, 98, 257-266.

(Manuscript received August 1, 1984; revision accepted for publication January 11, 1985.) 